\title{
Periodicity in Host Availability Does Not Account for Evolutionary Branching as Observed in Many Plant Pathogens: An Application to Gaeumannomyces graminis var. tritici
}

\author{
F. van den Berg, C. A. Gilligan, D. J. Bailey, and F. van den Bosch
}

First and fourth authors: Department of Biomathematics and Bioinformatics, Rothamsted Research, Harpenden, Hertfordshire AL5 2JQ, UK; second author: Department of Plant Sciences, University of Cambridge, Downing Street, Cambridge, CB2 3EA, UK; and third author: INRA-Agrocampus Rennes, UMR BiO3P, BP 35527, F-35653 Le Rheu Cedex, France.

Accepted for publication 18 June 2010.

\begin{abstract}
van den Berg, F., Gilligan, C. A., Bailey, D. J., and van den Bosch. F. 2010. Periodicity in host availability does not account for evolutionary branching as observed in many plant pathogens: An application to Gaeumannomyces graminis var. tritici. Phytopathology 100:1169-1175.

Periodicity in host availability is common in agricultural systems. Although it is known to have profound effects on plant pathogen abundance, the evolutionary consequences of periodicity for the pathogen population have not previously been analyzed. An epidemiological model incorporating periodic absence of the host crop is combined with the theory of adaptive dynamics to determine whether or not seasonality in

study is motivated and illustrated by the specific example of take-all disease of wheat, caused by the pathogen Gaeumannomyces graminis var. tritici, for which two coexisting but genetically distinct types and a tradeoff related to seasonality in host presence have been identified. Numerical simulations are used to show that a trade-off between the pathogen transmission rate and the survival of the pathogen between cropping seasons cannot account for the evolutionary branching observed in many pathogens. Model elaborations show that this conclusion holds for a broad range of putative mechanisms. Although the analysis is motivated and illustrated by the specific example of take-all of wheat, the results apply to a broad range of pathogens.
\end{abstract} host presence plays a role in the occurrence of evolutionary branching, leading to coexisting yet genetically distinct pathogen phenotypes. The

Agricultural ecosystems are characterized by seasonal crop dynamics, during which pathogen populations fluctuate in response to periodic changes in environmental variables and seasonal changes in the availability of susceptible crop hosts. The effect of such seasonality upon the evolution of pathogens has received little attention thus far. This is rather surprising because seasonality is known to have profound effects on the population dynamics of plant pathogens $(4,12,22,23,34)$ and, thus, is also likely to affect a pathogen's evolutionary dynamics through the very different selection pressures imposed on pathogens within an environment of fluctuating host availability.

In most models studying the evolution of virulence or pathogen aggressiveness, selection takes place in a continuously present host population $(2,15-20,37,38,40)$. Based on a trade-off between components of pathogen fitness, the main assumption of these models is that a high degree of pathogenicity leads to more rapid host death and, in turn, a reduction in the long-term survival of the pathogen (5). Evolutionary analysis shows that, for this class of models, the pathogen evolves to intermediate levels of pathogen aggressiveness, and that evolutionary branching, which would lead to closely related subgroups within the population, does not occur.

In systems with alternating presence and absence of the host, one could envisage an alternative trade-off. Although high aggressiveness increases the number of offspring during the crop growth season, which is advantageous in the short term, it can be

Corresponding author: F. van den Berg

E-mail address: femke.vandenberg@bbsrc.ac.uk

doi:10.1094/PHYTO-10-09-0282

(C) 2010 The American Phytopathological Society
Additional keywords: coexistence, seasonal trade-off. disadvantageous in the long term because high aggressiveness might be expected to be inversely correlated with a low survival between crop host growing seasons. For soilborne pathogens, a high aggressiveness, giving rise to increased production of offspring during the crop-growing season, may become disadvantageous in the longer term because a high resource use during the growing season leaves the pathogen with fewer resources to invest in, for example, resting spores for survival during the intercrop period due to the physiological constraint on the pathogen's total resource exploitation capacity. Although many soil pathologists do recognize this potential trade-off, few studies have attempted to quantify it $(1,11,30)$. A trade-off between parasitic pathogen success and saprotrophic survival has recently been identified and measured in Gaeumannomyces graminis var. tritici, the fungal pathogen that causes take-all disease in wheat (unpublished data).

In this article, we investigate the role of seasonality in the presence and absence of the host on evolutionary branching leading to the emergence of two types of coexisting yet genetically distinct phenotypes. We consider two phenotypes, one of which is relatively aggressive when the host is present but a poor survivor otherwise, whereas the other phenotype survives well in the absence of the host yet is not very aggressive when the host is present. The hypothesis that this trade-off gives rise to evolutionary branching is tested by combining an epidemiological model incorporating periodic host absence with the theory of adaptive dynamics. The sensitivity of the conclusions drawn from the basic model is tested by considering elaborations.

We address generic issues that have broad applicability to different pathogens while using a specific example to motivate and illustrate the analysis. Our motivation to study the specific case for the ubiquitous soilborne fungal pathogen of wheat, $G$. 
graminis var. tritici, comes from the observation that, for this pathosystem, two coexisting but genetically distinct pathotypes have been identified using molecular markers $(8,29)$; and because, for this system, a trade-off between parasitic pathogen success and saprotrophic survival has been quantified (unpublished data). Furthermore, heterokaryosis and parasexual recombination are believed to be rare in $G$. graminis, leading to clonally reproducing lines in which genetic variation arises by mutation $(26,31,33)$.

\section{MATERIALS AND METHODS}

The basic model. The model represents a crop-pathogen system with seasonal host growth and harvesting. Let $T$ be the length of one cycle and $\tau$ the period of this cycle in which the host is present $(0<\tau<T)$. We consider a model for the coupled changes in the availability of pathogen resource $(V)$ (e.g., crop roots or leaf tissue), pathogen densities during the crop-growing season for a resident strain $\left(P_{r}\right)$ and an invading strain $\left(P_{i}\right)$, and survival stage densities for a resident strain $\left(Q_{r}\right)$ and an invading strain $\left(Q_{i}\right)$. The model is given by

$$
\begin{aligned}
& \text { Crop growing period }(n T<t<n T+\tau) \quad \frac{\mathrm{d} V}{\mathrm{~d} t}=-\alpha V\left(\beta_{r} P_{r}+\beta_{i} P_{i}\right) \text {, } \\
& \frac{\mathrm{d} P_{r}}{\mathrm{~d} t}=\beta_{r} V P_{r}, \\
& \frac{\mathrm{d} P_{i}}{\mathrm{~d} t}=\beta_{i} V P_{i} \\
& \text { Crop free period }(n T+\tau<t<(n+1) T) \quad \frac{\mathrm{dQ}_{r}}{\mathrm{~d} t}=-\mu_{r} Q_{r} \text {, } \\
& \frac{\mathrm{dQ}_{i}}{\mathrm{~d} t}=-\mu_{i} Q_{i} \quad \text { with } \varepsilon_{j}=e^{-\mu_{j}(T-\tau)} j=r, i .
\end{aligned}
$$

in which the pathogen growth rates (equivalent to transmission rates) are given by $\beta_{r}$ and $\beta_{i}$ for the resident and invading strains, respectively, with values of 0 to $0.1 \mathrm{day}^{-1} ; \alpha$ is the resource use efficiency of the pathogen, assumed to be constant for resident and invading strains and set to $0.4 \mathrm{~m}^{2}$ (chosen arbitrarily). At the end of each cropping period, the pathogen population produces $\theta_{1}$ surviving stages per infection unit $\left[Q\left(n T+\tau^{+}\right)=\theta_{1} P\left(n T+\tau^{-}\right)\right.$and $\left.V\left(n T+\tau^{+}\right)=P\left(n T+\tau^{+}\right)=0\right]$. During this period of host absence, the pathogen survival stages have a constant death rate, $\mu$, resulting in crop-free survival probabilities $\varepsilon_{r}$ and $\varepsilon_{i}$ for resident and invading strains, respectively. At the beginning of each successive season, the host population starts at a fixed density,
$V\left(n T^{+}\right)=V_{0}$ with $0<V_{0}<K$ and $n=(1,2,3 \ldots)$. This resource replenishment resembles the planting of a crop. The pathogen lesion density at the beginning of the crop-growing season is determined by the density of survival stages surviving the crop free period, whereby each survival stage has a probability $\theta_{2}$, resulting in an infection $\left[P\left(n T^{+}\right)=\theta_{2} Q\left(n T^{-}\right)\right.$and $\left.Q\left(n T^{+}\right)=0\right]$.

The trade-off. The trait under evolution is the pathogen growth rate, $\beta_{j}$, and the associated trade-off specifies that a higher growth rate in the presence of the host, $\beta_{j}$, results in a lower pathogen survival probability in the absence of the host, $\varepsilon$ (Fig. 1A). This trade-off is given by

$$
\varepsilon_{j}=f_{1}\left(\beta_{j}\right)=\frac{A\left(\beta_{j}-\beta_{\max }\right)}{\beta_{j}-B}, j=r, i
$$

where $A$ and $B$ are shape parameters and $\beta_{\max }$ is the maximum pathogen growth rate. Given the strain specific growth rate, $\beta_{j}$, the associated constant pathogen survival probability, $\varepsilon$, is calculated from equation 2 . This constant survival probability, $\varepsilon_{j}$, leads to an exponential decline in the pathogen population with rate $\mu$. The strain-specific pathogen death rate during the crop-free period, $\mu_{j}$, is thus calculated by substituting equation 2 into the exponential function as given in equation 1 and subsequently solving for $\mu_{j}$.

Analytical analysis. For the basic model, it is possible to prove that the analytical results are general and, thus, independent of parameter choices. However, because of the strong mathematical nature of this analysis, we only summarize the findings from it and, instead, present the findings of the numerical simulation analysis. For the full analytical analysis, please refer to the Appendix.

Numerical simulation analysis. Consider a population of an annual crop with a monomorphic resident pathogen population. All offspring are phenotypically identical to the parents unless a genetic change occurs, leading to a change in offspring phenotype. Offspring with a phenotype that differs from the parent phenotype are called the invader phenotype. At this stage, phenotypic changes are assumed to be small and to affect one pathogen trait only. The simulations track the fate of invader phenotypes entering the resident population, whereby a sequence of successful invasions causes a directional change in pathogen phenotype. To implement the evolutionary dynamics, the range of the evolving parameter (i.e., the resident's growth rate, $\beta_{r}$ ) was divided into 50 equally spaced values in which each value or compartment represents a strain with a different phenotype. The initially monomorphic pathogen population resides in a randomly
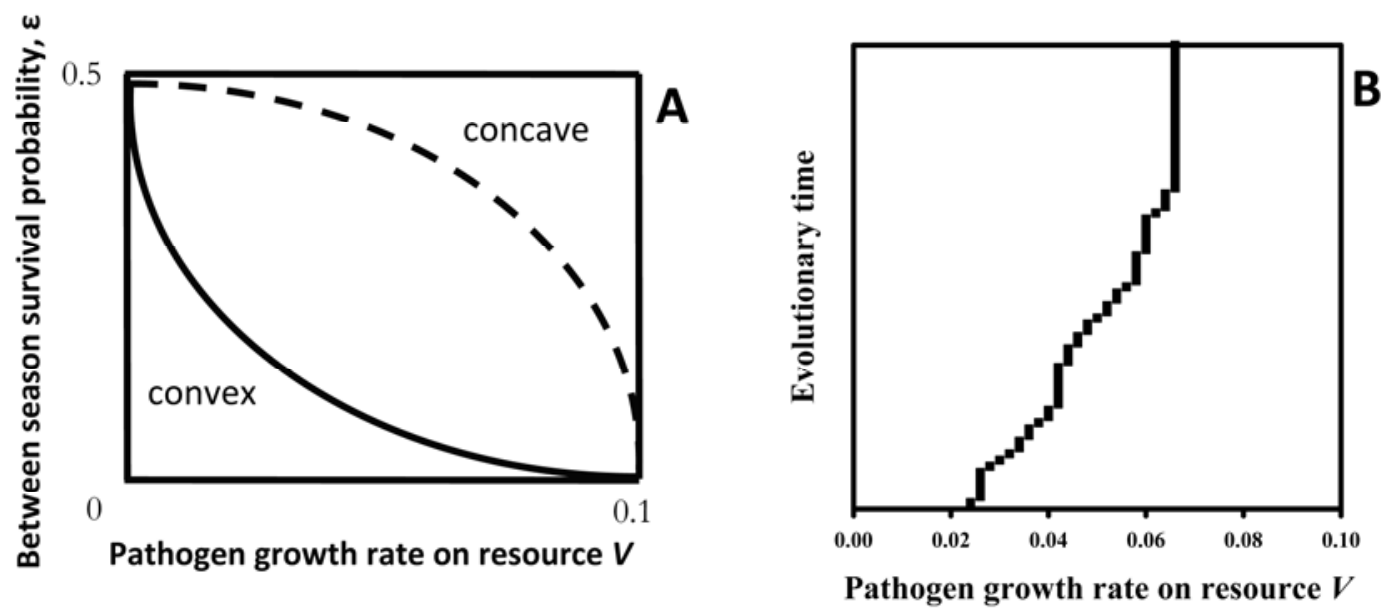

Fig. 1. A, Seasonal trade-off relationship between the pathogen survival probability in the intercrop period $\left(\varepsilon_{j}\right)$ and the pathogen growth rate $\left(\beta_{j}\right)$. Note that Bailey et al. (unpublished data) found a maximum inoculum survival probability of 0.53 and an average of 0.27 . B, Simulation of the evolutionary dynamics of the pathogen growth rate on resource $V$ in (evolutionary) time for the basic model (equation 1$)$ in the presence of the concave seasonal trade-off $(A=0.75, B=0.15$, and $\beta_{\max }=0.1$ ). The pathogen population has an initial growth rate, $\beta_{i}$, of 0.024 . 
chosen compartment. The arbitrary choice of initial value does not affect the results, which are independent of the initial conditions (analysis not shown). Subsequently, the evolutionary pathways are defined by the following rules. If a change in phenotype occurs, an invader phenotype develops such that the offspring is placed in a neighboring compartment with an equal probability of being on the left (i.e., smaller) or on the right (larger) of the parent or resident compartment. The new invader density is assumed to be very small compared with the original resident population density. The system in the presence of the invader is subsequently run until equilibrium is reached (i.e., the long-term average host and pathogen densities approach constant values) with three potential outcomes: (i) unsuccessful invasion by the new phenotype offspring, in which the system has reverted to its old state (i.e., only the resident strain compartment contains individuals); (ii) successful invasion in which the invader phenotype out-competes the resident strain and itself becomes the new resident phenotype (i.e., only the invader strain compartment contains individuals); or (iii) successful invasion in which the invader co-exists with the resident strain, leading to evolutionary branching (i.e., both the resident and invader strain compartments contain individuals). The evolutionary process is continued until no changes are observed for 20 consecutive mutation events, at which point it is assumed that the evolutionary end point has been reached. Note that we assume that the system has reverted to equilibrium (this assumption is relaxed later) before a new mutation event is allowed to occur and, thus, that the evolutionary processes occur on a much larger time scale than the ecological processes (i.e., the time scale used for the integration of the population dynamics of equations 1 and 2).

For the numerical simulations, we use the following parameter values: the season length is 150 days; at the start of each new season, the resource density, $V_{0}$, is $5 \mathrm{~m}^{-2}$ (unless stated otherwise); the initial resident parasite density, $P_{r}$, is $0.1 \mathrm{~m}^{-2}$ for the first season and subsequently depends on the resident's survival in the absence of the host; and the invader density, $P_{i}$, is $0.01 \times P_{r} \mathrm{~m}^{-2}$ (at time of invasion) at the beginning of the season in which the invader strain first appears and, subsequently, depends on the invader's survival in the absence of the host. Furthermore, $\theta_{1}=$ 10 , resulting in each lesion producing 10 survival stages; and $\theta_{2}=$ 0.1 , resulting in a probability of 0.1 for a survival stage to cause an infection at the beginning of the crop growing season. Finally, the resource use efficiency of the parasite, $\alpha$, is $0.4 \mathrm{~m}^{2}$ and the parasite growth rate on resource $V, \beta$, is 0 to 0.1 day $^{-1}$. For illustrative purposes, default parameters are chosen such that they reflect population densities that are realistic for take-all on wheat $(7,10)$ but the parameter ranges studied are wide enough to encompass a range of different host-pathogen systems.

Seasonal trade-off with additional elaborations. To study the sensitivity of the qualitative results for the basic model (equation 1), together with the seasonal trade-off (equation 2), to changes in the model structure (structural stability), we consider five model elaborations (I to V; Table 1). For each of the model elaborations, only the within-season dynamics are altered; for the dynamics of the crop free period and the trade-off, see equations 1 and 2 . In this section, only newly introduced parameters and variables are discussed; for all other variable and parameter descriptions and values, please refer to the basic model. The five model elaborations considered are as follows.

I. Resource growth model. Crops are often grown from seed, leading to a sharp increase in host tissue or resource availability from the time of germination at the start of the growing season until crop maturation. In ecology, this type of population growth is often represented by a logistic equation, where the rate of reproduction, $r$, is proportional to the existing population, $V$, and the total amount of available resources, $K$, otherwise known as the carrying capacity. The within-season dynamics of the resource growth model can thus be formalized by

$$
\begin{aligned}
\frac{\mathrm{d} V}{\mathrm{~d} t} & =r V\left(1-\frac{V}{K}\right)-\alpha V\left(\beta_{r} P_{r}+\beta_{i} P_{i}\right), \\
\frac{\mathrm{d} P_{r}}{\mathrm{~d} t} & =\beta_{r} V P_{r}, \\
\frac{\mathrm{d} P_{i}}{\mathrm{~d} t} & =\beta_{i} V P_{i} .
\end{aligned}
$$

Here, the value of the intrinsic rate of resource growth, $r$, is 0.0001 to $10 \mathrm{day}^{-1}$ and resource carrying capacity, $K$, is set to 15 $\mathrm{m}^{-2}$.

II. Functional response model. When resource availability is high, the pathogen resource uptake is likely to become saturated. Such a functional saturation response can be represented by a Monod function, leading to

$$
\begin{aligned}
\frac{\mathrm{d} V}{\mathrm{~d} t} & =-\alpha \frac{V}{1+\eta V}\left(\beta_{r} P_{r}+\beta_{i} P_{i}\right), \\
\frac{\mathrm{d} P_{r}}{\mathrm{~d} t} & =\beta_{r} \frac{V}{1+\eta V} P_{r}, \\
\frac{\mathrm{d} P_{i}}{\mathrm{~d} t} & =\beta_{i} \frac{V}{1+\eta V} P_{i} .
\end{aligned}
$$

Here, $\eta$ is a shape parameter with a value of 0.001 to 5 .

III. Pathogen loss term model. The basic model assumes that no pathogen death occurs during the host growing season; however, here we introduce a pathogen death rate during the crop growing season. The pathogen loss term model thus reads

$$
\begin{aligned}
\frac{\mathrm{d} V}{\mathrm{~d} t} & =-\alpha V\left(\beta_{r} P_{r}+\beta_{i} P_{i}\right), \\
\frac{\mathrm{d} P_{r}}{\mathrm{~d} t} & =\beta_{r} V P_{r}-\omega P_{r}, \\
\frac{\mathrm{d} P_{i}}{\mathrm{~d} t} & =\beta_{i} V P_{i}-\omega P_{i} .
\end{aligned}
$$

where $\omega$ is the pathogen death rate, with a value of 0.0001 to 0.03 day $^{-1}$.

IV. Primary inoculum model. Take-all disease on cereal roots spreads through infections arising from both primary and secondary inoculum. Primary infection is initiated from a declining reservoir of inoculum, $X$, whereas secondary infections arise from transmission through mycelial contact between infected, $P$, and susceptible, $V$, host tissue. When taking such primary infection into account, the model is given by

$$
\begin{aligned}
\frac{\mathrm{d} V}{\mathrm{~d} t} & =-\alpha V\left(\beta_{r} P_{r}+\beta_{i} P_{i}\right)-\alpha V\left(\beta_{r} \gamma X_{r}+\beta_{i} \gamma X_{i}\right), \\
\frac{\mathrm{d} P_{r}}{\mathrm{~d} t} & =\beta_{r} V P_{r}+\beta_{r} \gamma X_{r} V, \\
\frac{\mathrm{d} P_{i}}{\mathrm{~d} t} & =\beta_{i} V P_{i}+\beta_{i} \gamma X_{i} V, \\
\frac{d X_{r}}{d t} & =-\delta X_{r}, \\
\frac{d X_{i}}{d t} & =-\delta X_{i} .
\end{aligned}
$$

Here, $\delta$ represents the inoculum decay rate $\left(0.001\right.$ to 5 day $\left.^{-1}\right)$ and $\gamma$ is a conversion factor (2; dimensionless). In this case, $P\left(n T^{+}\right)=$ 0 and $X\left(n T^{+}\right)=\theta_{3} Q\left(n T^{-}\right)$, with $\theta_{3}=0.1$ resulting in a probability of 0.1 for a survival stage to produce viable primary inoculum at the beginning of the crop growing season.

V. Two antagonism models. Antagonism-pathogen growth suppression by other organisms such as bacteria or fungi-is a key feature in soil pathosystems $(13,21,36)$ but little is known about the mode of action of these antagonists. Here, we compare two very contrasting modes of action. 
(i) An antagonist directly affects the pathogen. In this case, the antagonist, $A$, acts as a predator or hyperparasite to the pathogen and, thereby, reduces the pathogen growth rate

$$
\begin{aligned}
\frac{\mathrm{d} V}{\mathrm{~d} t} & =-\alpha V\left(\beta_{r} P_{r}+\beta_{i} P_{i}\right), \\
\frac{\mathrm{d} P_{r}}{\mathrm{~d} t} & =\beta_{r} V P_{r}-\beta_{A} P_{r} A, \\
\frac{\mathrm{d} P_{i}}{\mathrm{~d} t} & =\beta_{i} V P_{i}-\beta_{A} P_{i} A, \\
\frac{d A}{d t} & =\beta_{A} A\left(P_{r}+P_{i}\right)-\omega A .
\end{aligned}
$$

Here, $\beta_{A}$ is the antagonist attack rate $\left(0\right.$ to 0.03 day $\left.^{-1}\right)$ and $\omega$ is the antagonist death rate $\left(0.1\right.$ day $\left.^{-1}\right)$. The survival probability of the antagonist in the absence of the crop is set to $0.01 \mathrm{day}^{-1}$ and the initial antagonist density, $A_{0}$, is set to $0.1 \mathrm{~m}^{-2}$.

(ii) An antagonist overgrows the resource. In this case, the antagonist colonizes the resource used by the pathogen at rate $\rho$. Resource material that is colonized by the antagonist is less accessible to the pathogen, resulting in a fractional reduction of the pathogen infection rate, $\sigma$, of 0.25 on colonized resource material $C$. When the antagonist dies, the previously colonized resource becomes fully accessible to the pathogen again and, hence, an antagonist death rate of $\omega$ moves resource from class $C$ to class $V$ at the same rate of $\omega$. Note that the antagonist does not feed directly on either the resource or the pathogen but, instead, lives on the by-products associated with the pathogen infection process, whereby root cells are damaged and chemicals or

\begin{tabular}{|c|c|c|c|c|c|}
\hline \multirow[b]{2}{*}{ Model $^{\mathrm{b}}$} & \multicolumn{5}{|c|}{ Model equations } \\
\hline & $\begin{array}{l}\text { Equation 1: } \\
\text { Resource }\end{array}$ & $\begin{array}{c}\text { Equation 2: } \\
\text { Parasite } 1\end{array}$ & $\begin{array}{c}\text { Equation } 3 \text { : } \\
\text { Parasite } 2\end{array}$ & $\begin{array}{c}\text { Equation 4: } \\
\text { Various }\end{array}$ & $\begin{array}{c}\text { Equation 5: } \\
\text { Various }\end{array}$ \\
\hline Basic $^{c}$ & $\frac{d V}{d t}=-\alpha V\left(\beta_{r} P_{r}\right.$ & $\frac{d P_{r}}{d t}=\beta_{r} V P$ & $\frac{d P_{i}}{d t}=\beta_{i} V P_{i}$ & & \\
\hline
\end{tabular}
compounds are released; thus, we do not explicitly keep track of the antagonist density. The model is then given by

TABLE 1. Model scenarios ${ }^{\mathrm{a}}$

Resource growth $^{\mathrm{d}}$

Functional response $\mathrm{e}^{\mathrm{a}}$

Parasite

loss term ${ }^{\mathrm{f}}$

Primary

inoculumg

$-\alpha V\left(\beta_{r} \gamma X_{r}+\beta_{i} \gamma X_{i}\right)$

$+\beta_{r} \gamma X_{r} V$

$+\beta_{i} \gamma X_{i} V$ $\cdot\left(\frac{1}{1+\eta V}\right) \cdot\left(\frac{1}{1+\eta V}\right)$

$-\omega P_{r} \quad-\omega P_{i}$ $\frac{d X_{r}}{d t}=-\delta X_{r}$

$\frac{d A}{d t}=\beta_{A} A\left(P_{r}+P_{i}\right)-\omega A$
Antagonist: directly affecting parasite ${ }^{\mathrm{h}}$

$$
-\beta_{A} P_{r} A \quad-\beta_{A} P_{i} A
$$

$$
\begin{aligned}
+\beta_{r}(1-\sigma) C P_{r}+\beta_{i}(1-\sigma) C P_{i} \quad \frac{d C}{d t}= & \rho V C-\omega C \\
& -\alpha(1-\sigma) C\left(\beta_{r} P_{r}+\beta_{i} P_{i}\right)
\end{aligned}
$$

overgrowing resource $\mathrm{e}^{\mathrm{i}}$

$$
-\rho V C+\omega C
$$

${ }^{a}$ Basic model (equation 1) is used as a reference, whereas the individual rows indicate which terms need to be added to which equation of the basic model to create models I to V. Parameters and their default values are explained in the footnotes. For all model extensions, a wide parameter range was chosen.

${ }^{\mathrm{b}}$ For all the models described in this table, the season length is 150 days; the initial resource density, $V_{0}$, is $5 \mathrm{~m}^{-2}$ (unless stated otherwise) and stays constant over seasons; the initial resident parasite density, $P_{r}$, is $0.1 \mathrm{~m}^{-2}$ for the first season and subsequently depends on the resident's survival in the absence of the host; and the initial invader density, $P_{i}$, is $0.01 \times P_{r} \mathrm{~m}^{-2}$ (at time of invasion) for the first season after invasion and subsequently depends on the invader's survival in the absence of the host. Furthermore, $\theta_{1}=10$, resulting in each lesion producing 10 survival stages, and $\theta_{2}=0.1$, resulting in a probability of 0.1 for a survival stage to cause an infection at the beginning of the crop growing season.

${ }^{c}$ Parameters: $\alpha=$ resource use efficiency of the parasite $\left(0.4 \mathrm{~m}^{2}\right.$; chosen arbitrarily); $\beta=$ parasite growth rate on resource $V\left(0\right.$ to 0.1 day $\left.{ }^{-1}\right)$. Default parameters for the basic model are chosen such that the parasite densities are realistic for take-all on wheat $(6,9)$.

d Parameters: $r=$ intrinsic rate of resource growth $\left(0.0001\right.$ to 10 day $\left.^{-1}\right)$ and $K=$ resource carrying capacity $\left(15 \mathrm{~m}^{-2}\right)$.

e Parameter: $\eta=$ shape parameter (0.001 to 5; dimensionless).

f Parameter: $\omega=$ parasite death rate $\left(0.0001\right.$ to 0.03 day $\left.^{-1}\right)$.

g Variable: $X_{r}$ and $X_{m}=$ densities of resident's and invader's primary inoculum $\left(\mathrm{m}^{-2}\right)$. Parameters: $\delta=$ inoculum decay rate $\left(0.001-5\right.$ day $\left.{ }^{-1}\right)$ and $\gamma=$ conversion factor (2; dimensionless). In this case, $P\left(n T^{+}\right)=0$ and $X\left(n T^{+}\right)=\theta_{3} Q\left(n T^{-}\right)$, with $\theta_{3}=0.1$ resulting in a probability of 0.1 for a survival stage to produce viable primary inoculum at the beginning of the crop growing season.

h Variable: $A=$ antagonist density $\left(\mathrm{m}^{-2}\right)$. Parameters: $\beta_{A}=$ antagonist attack rate $\left(0\right.$ to 0.03 day $\left.^{-1}\right)$ and $\omega=$ antagonist death rate $\left(0.1\right.$ day $\left.{ }^{-1}\right)$. Survival probability of the antagonist in the absence of the crop is 0.01 day $^{-1}$. Initial antagonist density, $A_{0}$, is $0.1 \mathrm{~m}^{-2}$.

${ }^{\text {i }}$ Variable: $C=$ colonized resource density $\left(\mathrm{m}^{-2}\right)$. Parameters: $\rho=$ antagonist colonization rate $\left(0.1\right.$ day $\left.^{-1}\right) ; \omega=$ antagonist tissue death rate $\left(0.1\right.$ day $\left.{ }^{-1}\right)$; $\sigma=$ fractional reduction in the parasite infection rate due to antagonist colonization $\left(0.25\right.$; dimensionless). Initial density of colonized resource, $C_{0}$, is $0.1 \mathrm{~m}^{-2}$. 


$$
\begin{aligned}
\frac{\mathrm{d} V}{\mathrm{~d} t} & =-\alpha V\left(\beta_{r} P_{r}+\beta_{i} P_{i}\right)-\rho V C+\omega C, \\
\frac{\mathrm{d} P_{r}}{\mathrm{~d} t} & =\beta_{r} V P_{r}+\beta_{r}(1-\sigma) C P_{r}, \\
\frac{\mathrm{d} P_{i}}{\mathrm{~d} t} & =\beta_{i} V P_{i}+\beta_{i}(1-\sigma) C P_{i}, \\
\frac{d C}{d t} & =\rho V C-\omega C-\alpha(1-\sigma) C\left(\beta_{r} P_{r}+\beta_{i} P_{i}\right) .
\end{aligned}
$$

where $\rho$ is $0.1 \mathrm{day}^{-1}, \omega$ is $0.1 \mathrm{day}^{-1}$, and the initial density of colonized resource, $C_{0}$, is $0.1 \mathrm{~m}^{-2}$.

\section{RESULTS}

An analytical analysis of the basic model shows that the evolution of the pathogen growth rate is such that it maximizes the pathogen's basic reproduction number, $R_{0}$, leading to competitive exclusion (Appendix). These results are independent of the individual parameter choices. Thus, evolutionary branching leading to two coexisting yet genetically distinct pathotypes is not possible, despite the presence of seasonality in host availability. The proof is exemplified by the basic model but is equally valid for model elaborations I to III. For the remaining model elaborations, the additional equations make the analytical analysis rather complex; therefore, for these models, we only performed numerical simulation analyses.

The simulation analysis results of the basic model agree with those found by analytical analysis, and the results for the basic model in the presence of a concave seasonal trade-off are shown in Figure 1B. The simulation was started with a monomorphic resident population and a pathogen growth rate of $\beta=0.024$. In this case, the population is (over evolutionary time) continuously replaced by another population with a slightly higher pathogen growth rate until the pathogen growth rate reaches $\beta=0.064$ which, for this particular set of parameter values, represents the evolutionary end point. The population remains monomorphic throughout. These results remain unchanged for different initial pathogen growth rates, $\beta$, as well as for alternative parameter values. We conclude that, for the basic model, a concave seasonal trade-off between the pathogen growth rate and its survival rate between crops is insufficient to explain evolutionary branching into coexisting and closely related phenotypes. The results remain unchanged when the concave trade-off is replaced by a convex seasonal trade-off (data not shown).

None of the model extensions led to a qualitative difference in the results (i.e., as for the basic model, no evolutionary branching occurs but, for several of the model elaborations, a quantitative difference in results is found). When the model is extended to include a pathogen loss term during the growing season or an antagonist that directly affects the pathogen, the evolutionary end point is shifted to higher pathogen growth rates (Fig. 2). More specifically, the figure shows that, when the within-crop growing season death rates of the pathogen are increased, more aggressive pathogen strains evolve. The main conclusion is that a seasonal trade-off alone cannot explain evolutionary branching into two coexisting phenotypes.

Additional simulations show that the qualitative conclusions are not sensitive to the assumption that mutations are small and occur rarely enough for the system to have reverted to equilibrium before the next mutation event (results not shown). In these simulations, we kept track of 50 pathogen equations, 1 for each compartment. At the start of the simulation, only one randomly chosen strain is present. We then assume that, at the start of each season, each strain has a fixed probability to mutate, offspring either being moved to the neighboring compartments in the case of small mutations or to a random compartment distant from the parent compartment in the case of large mutations. Furthermore, a population is assumed to have died out when its density falls below a certain threshold value. These simulations show that, for the basic model, the pathogen population converges to a growth rate of $\beta=0.064$ (cf. Fig. 1). Although some strains with very similar growth rates remain present in the population due to the continuous mutation, their densities are very low in comparison. All other more extreme strains go extinct, confirming our previous results that evolutionary branching leading to the coexistence of two phenotypes cannot be explained by the presence of seasonality in host availability. Note that the second part of the Appendix provides a general analytical proof for the fact that the results are independent of the size of the mutation steps.

\section{DISCUSSION}

There are many examples of coexisting but genetically distinct pathotypes cited in the literature $(9,24,25)$. The evolution of such pathotypes is not well understood but the morphological and genetic similarities between the individuals suggest that they have evolved from a common ancestor. It is well known that, for speciation to occur, certain requirements need to be met (i.e., the population needs to be interbreeding and there has to be natural selection, reproductive isolation, and an accumulation of genetic differences) (35). Thus far, the study by Gudelj et al. $(24,25)$ is the only one to investigate which ecological mechanisms could be the main driving force behind the evolution of such coexisting but genetically distinct pathotypes. In their study, Gudelj et al. $(24,25)$ investigated the potential of host specialization as a selection pressure resulting in evolutionary branching, leading to sibling plant pathogens. They showed that, whereas host specialization could explain the evolution of sibling pathogens that are completely host-specialized, it cannot explain the evolution of sibling pathogens with (partly) shared host ranges.

In this article, we have studied whether or not an ecological mechanism such as seasonality in host availability, in combination with a trade-off between life-cycle components that operate
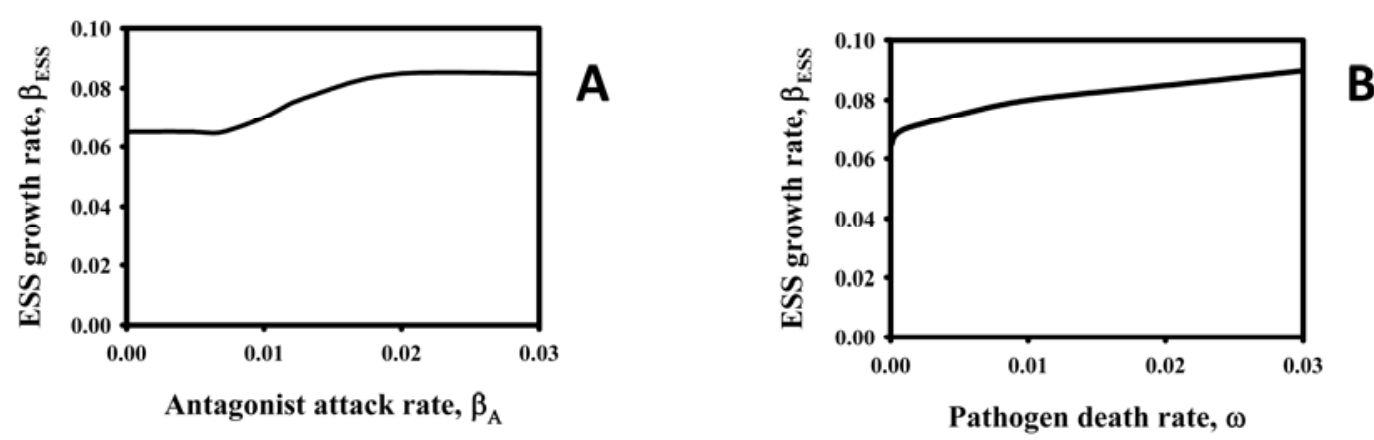

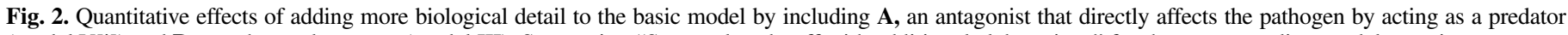
(model V[i]) and B, a pathogen loss term (model III). See section "Seasonal trade-off with additional elaborations" for the corresponding model equations. 
in different parts of the cropping cycle, can result in similar evolutionary dynamics, and whether it could possibly also explain the occurrence of sibling pathogens that are not completely specialized. An initial hypothesis was that the seasonal dynamics could provide the ecological distinction required for speciation, resulting in one type with a high within-season aggressiveness but low between-season survival and the other with low within-season aggressiveness but high between-season survival. This idea was further motivated by data found for take-all of wheat, which showed a trade-off between within-season aggressiveness and between-season survival (unpublished data), and further supported by evidence for the existence of similar trade-offs in other soilborne pathosystems such as Rhynchosporium secalis (1) and Cochliobolus heterostrophus (11).

We have shown both by analytical analysis (Appendix) and simulations (Fig. 1) that a seasonal trade-off between the withinseason transmission rate and the between-season survival rate does not select for evolutionary branching into two distinct coexisting phenotypes. Importantly, extending the basic model (equations 1 and 2) to include more epidemiological processes, such as resource growth and antagonism (seasonal trade-off with additional elaborations), does not alter this conclusion. This suggests that the conclusions from the simple and parametersparse basic model in this article are robust against a wide range of model extensions.

Discussions among plant pathologists on the matter of evolutionary branching leading to coexisting generalist sibling pathogens always result in an array of suggested hypotheses. However, two of the most convincing hypotheses have now been falsified: (i) seasonality as defined in this study cannot result in speciation and (ii) resource specialization as defined by Gudelj et al. $(24,25)$ can only explain one type of speciation. Therefore, there must be other ecological mechanisms responsible for the existence of sibling pathogens that share host or niche ranges. As suggested by Gudelj et al. (24,25), an example of such ecological mechanisms could be a trade-off between the amount of rain-dispersed and wind-dispersed spores produced. Other examples are a trade-off between the amount of sexual and asexual spores produced, a trade-off between the amount of spores produced leading to secondary infections and the amount of free-living resting spores, or a trade-off in the efficiency with which the pathogen uses different resources within a single host. We are currently testing some of these alternative hypotheses.

Generalizations and management implications. We have mainly focussed on soilborne pathogens in this article. However, a trade-off between pathogen aggressiveness and the intercrop survival probability also exists for airborne pathogens, which suggests that the results are relatively generic and apply to a wide range of different types of host pathosystems. In the airborne fungal pathogen Phaeosphaeria nodorum, for example, transmission within the crop season occurs by the production of spores in fungal lesions on leaves, whereas survival between growing seasons is to an important extent on crop seed (3). Mycelium growth rate, a measure of pathogen aggressiveness, is correlated with the size of the lesions developing on the leaf which, in turn, is correlated with spore production (28). Mycelium growth rate is also correlated with the severity of seed infestation, where severe infestation reduces seed size and viability (14), which significantly reduces the probability that a seed will be used as planting material, germinate, and develop into an infected host plant $(14,27)$. As for the soilborne systems, this implies that there may be a trade-off between pathogen aggressiveness and the intercrop survival probability.

Components or environmental factors that affect within-season pathogen survival seem to select for more aggressive pathogen strains (Fig. 2). For example, Figure 2B suggests that efficient biological control with an antagonist might select for strains with a higher transmission rate. van den Bosch et al. (39) similarly found that certain virus control strategies can lead to the selection of virus strains that build up a high within-plant virus titer. This has major implications for disease management because, even though these disease control methods might result in a large increase in the density of healthy plants in a crop, the resulting selection of more harmful parasite strains is a real possibility leading to unexpected yield loss. Therefore, the balance between these two factors ought to be taken into careful consideration when developing disease management strategies.

\section{APPENDIX: MATHEMATICAL PROOF FOR THE ABSENCE OF EVOLUTIONARY BRANCHING}

This appendix provides mathematical proof for the fact that the systems studied in this paper are always subject to competitive exclusion whereby the only strain remaining is the one with the highest value of $R_{0}$ (number of life-time offspring by a pathogen entering a virgin environment). The proof is exemplified by the basic model, but is equally valid for model elaborations I to III. For the remaining model elaborations, the additional equations make the analytical analysis rather complex, so for these models analysis is done by numerical simulations only.

The basic reproduction number, $\boldsymbol{R}_{\mathbf{0}}$. The dynamics of the disease-free hosts is given by $V^{*}\left(n T^{+}\right)=V_{0}$ and $d V^{*}(t) / d f=f\left(V^{*}(t)\right.$, $0)$ if $n T^{+}<t<n T+t^{-} ; V^{*}(t)=0$ if $n T+t^{+}<t<(n+1) T^{-}$and $P(t)$ $=Q(t)=0$ throughout the year. To derive $R_{0}$ the system is linearized near this disease-free state, resulting in

$$
\begin{aligned}
& \frac{d P}{d t}=\sigma_{1}(t) P-\eta_{1}(t) P \\
& \frac{d Q}{d t}=\sigma_{2}(t) Q-\eta_{2}(t) Q
\end{aligned}
$$

where $\sigma_{1}(t), \sigma_{2}(t), \eta_{1}(t)$, and $\eta_{2}(t)$ are nonnegative T-periodic functions given by

$$
\left.\begin{array}{l}
\left.\begin{array}{l}
\sigma_{1}(t)=\beta V^{*}(t) \\
\eta_{1}(t)=0 \\
\sigma_{2}(t)=0 \\
\eta_{2}(t)=\mu
\end{array}\right\} \text { for } n T^{+}<t<n T+\tau^{-}
\end{array}\right\} \text {for } n T+\tau^{+}<t<(n+1) T^{-} .
$$

Furthermore, $Q\left(n T+t^{+}\right)=P\left(n T+t^{-}\right)$and $P\left(n T^{+}\right)=Q\left(n T^{-}\right)$. Bacaer and Guernaoui (6) showed that for a system such as equation A1,

$$
R_{0}=\frac{\int_{0}^{\tau} \sigma_{1}(t) d t+\int_{\tau}^{T} \sigma_{2}(t) d t}{\int_{0}^{\tau} \eta_{1}(t) d t+\int_{\tau}^{T} \eta_{2}(t) d t}
$$

And we conclude that for our model equations 1

$$
R_{0}=\frac{\beta \bar{V}^{*} \tau}{\mu(T-\tau)} \text { with } \bar{V}^{*}=\frac{1}{\tau} \int_{0}^{\tau} V^{*}(t) d t
$$

$\mathbf{R}_{0}$ maximization leads to competitive exclusion. The following proof is an adaptation of the one given, e.g., by Nowak and Sigmund (32), for the case without seasonality. Given that $V(t)=0$ for $n T+t<t<(n+1) T$, the original nonlinear model can also be written as

$$
\frac{d Z_{r}}{d t}=\beta_{r} Z_{r} V-B_{r}(t) Z_{r}, \quad \frac{d Z_{i}}{d t}=\beta_{i} Z_{i} V-B_{i}(t) Z_{i}
$$

So

$$
\frac{1}{\beta_{r} Z_{r}} \frac{d Z_{r}}{d t}-\frac{1}{\beta_{i} Z_{i}} \frac{d Z_{i}}{d t}=-\frac{B_{r}(t)}{\beta_{r}}+\frac{B_{i}(t)}{\beta_{i}} .
$$


Here subscripts ' $r$ ' and ' $i$ ' represent the resident and invader strain, respectively. Assume $R_{0}{ }^{(i)}>R_{0}{ }^{(r)}>1$. Integrating and letting $t$ tend to infinity, we find

$$
\begin{aligned}
\frac{1}{\beta_{r}} \log \left(\frac{Z_{r}(t)}{Z_{r}(0)}\right)-\frac{1}{\beta_{i}} \log \left(\frac{Z_{i}(t)}{Z_{i}(0)}\right)= & -\frac{1}{\beta_{r}} \int_{0}^{t} B_{r}(u) d u+\frac{1}{\beta_{i}} \int_{0}^{t} B_{i}(u) d u \\
& \frac{t}{T}\left(-\frac{1}{\beta_{r}} \int_{0}^{T} B_{r}(u) d u+\frac{1}{\beta_{i}} \int_{0}^{T} B_{i}(u) d u\right), \\
& \frac{t}{T}\left(-\frac{1}{R_{0}^{(r)}}+\frac{1}{R_{0}^{(i)}}\right) \int_{0}^{T} V^{*}(u) d u, \\
& \rightarrow-\infty .
\end{aligned}
$$

So either $Z_{i}(t) \rightarrow \infty$ or $Z_{r}(t) \rightarrow 0$ as $t \rightarrow \infty$. Since $Z_{i}(t) \rightarrow+\infty$ impossible, $Z_{r}(t) \rightarrow 0$ is the only possible solution, leading to "competitive exclusion". The only strain remaining is the one with the highest value of $R_{0}$ and evolutionary branching leading to coexisting yet genetically distinct phenotypes is thus not possible.

\section{ACKNOWLEDGMENTS}

Rothamsted Research receives support from the Biotechnology and Biological Sciences Research Council (BBSRC) of the United Kingdom. C. A. Gilligan gratefully acknowledges support of a BBSRC Professional Fellowship. We thank N. Cunniffe, M. Gosme, P. Lucas, and A. Sarniguet for their useful discussions during the course of the work.

\section{LITERATURE CITED}

1. Abang, M. M., Baum, M., Ceccarelli, S., Grando, S., Linde, C. C., Yahyaoui, A., Zhan, J., and McDonald, B. A. 2006. Differential selection on Rhynchosporium secalis during parasitic and saprophytic phases in the barley scald disease cycle. Phytopathology 96:1214-1222.

2. Agnew, P., and Koella, J. C. 1997. Virulence, parasite mode of transmission, and host fluctuating asymmetry. Proc. R. Soc. London B 264:915 .

3. Agrios, G. N. 2005. Plant Pathology. Elsevier Academic Press, Amsterdam.

4. Altizer, S., Dobson, A., Hosseini, P., Hudson, P., Pascual, M., and Rohani, P. 2006. Seasonality and the dynamics of infectious diseases. Ecol. Lett. 9:467-484.

5. Anderson, R. M., and May, R. M. 1981. The population dynamics of microparasites and their invertebrate hosts. Phil. Trans. R. Soc. London B 291:451:524.

6. Bacaer, N., and Guernaoui, S. 2006. The epidemic threshold of vectorborne diseases with seasonality. J. Math. Biol. 53:421-436.

7. Bailey, D. J., Paveley, N., Pillinger, C., Foulkes, J., Spink, J., and Gilligan, C. A. 2005. Epidemiology and chemical control of take-all on seminal and adventitious roots of wheat. Phytopathology 95:62-68.

8. Bateman, G. L., Ward, E., Hornby, D., and Gutteridge, R. J. 1997. Comparisons of isolates of the take-all fungus, Gaeumannomyces graminis var. tritici, from different cereal sequences using DNA probes and non-molecular methods. Soil Biol. Biochem. 29:1225-1232.

9. Brasier, C. M. 1987. The dynamics of fungal speciation. In: Evolutionary Biology of the Fungi. A. D. M. Rayner, C. M. Brasier, and D. Moore, eds. Cambridge University Press, Cambridge.

10. Brassett, P. R., and Gilligan, C. A. 1989. Fitting of simple models for field disease progress data for the take-all fungus. Plant Pathol. 38:397-407.

11. Carson, M. L. 1998. Aggressiveness and perennation of isolates of Cochliobolus heterostrophus from North Carolina. Plant Dis. 82:10431047.

12. Caswell, H. 2001. Matrix Population Models, 2nd ed. Sinauer Associates Inc., Sunderland, MA.

13. Cook, R. J. 1981. Evidence that take-all decline does not result from loss of virulence in the population of Gaeumannomyces-graminis var. tritici. (Abstr.) Phytopathology 71:211.

14. Cunfer, B. M., and Johnson, J. W. 1981. Relationship of glume blotch symptoms on wheat heads to seed infection by Septoria nodorum. Trans.
Br. Mycol. Soc. 76:205-211

15. Dieckmann, U., Metz, J. A. J., Sabelis, M. W., and Sigmund, K. 2002. Cambridge Studies in Adaptive Dynamics. Adaptive Dynamics of Infectious Diseases: In Pursuit of Virulence Management: Cambridge University Press, International Institute for Applied Systems Analysis.

16. Ebert, D., and Herre, E. A. 1996. The evolution of parasitic diseases. Parasitol. Today 12:96-101.

17. Ewald, P. W. 2004. Evolution of virulence. Infect. Dis. Clin. N. Am. 18:1

18. Frank, S. A. 1996. Models of parasite virulence. Q. Rev. Biol. 71:37-79.

19. Gandon, S, Mackinnon, M. J., Nee, S., and Read, A. F. 2001. Imperfect vaccines and the evolution of pathogen virulence Nature 414:751-756.

20. Gandon, S., and Michalakis, Y. 2000. Evolution of parasite virulence against qualitative or quantitative host resistance. Proc. R. Soc. London B 267:985-990.

21. Gibson, G. J., Gilligan, C. A., and Kleczkowski, A. 1999. Predicting variability in biological control of a plant-pathogen system using stochastic models. Proc. R. Soc. London B 266:1743-1753.

22. Gubbins, S., and Gilligan, C. A. 1997. Biological control in a disturbed environment. Philos. Trans. R. Soc. London B 352:1935-1949.

23. Gubbins, S., and Gilligan, C. A. 1997. Persistence of host-parasite interactions in a disturbed environment. J. Theor. Biol. 188:241-258.

24. Gudelj, I., Fitt, B. D. L., and van den Bosch, F. 2004. Evolution of sibling fungal plant pathogens in relation to host specialization. Phytopathology 94:789-795.

25. Gudelj, I., van den Bosch, F., and Gilligan, C. A. 2004. Transmission rates and adaptive evolution of pathogens in sympatric heterogeneous plant populations. Proc. R. Soc. London B 271:2187-2194.

26. Harvey, P. R., Langridge, P., and Marshall, D. R. 2001. Genetic drift and host-mediated selection cause genetic differentiation among Gaeumannomyces graminis populations infecting cereals in southern Australia. Mycol. Res. 105:927-935.

27. Hewett, P. D. 1975. Septoria nodorum on seedlings and stubble of winter wheat. Trans. Br. Mycol. Soc. 65:7-18.

28. Jeger, M. J., Gareth Jones, D., and Griffiths, E. 1984. Sporulation of Septoria nodorum (and S. tritici) on spring wheat cvs. Kolibri and Maris Butler in relation to growth stage, plant part and time of season. Ann. Appl. Biol. 104:31-39.

29. Lebreton, L., Lucas, P., Dugas, F., Guillern, A.-Y., Schoeny, A., and Sarniguet, A. 2004. Changes in population structure of the soilborne fungus Gaeumannomyces graminis var. tritici during continuous wheat cropping. Environ. Microbiol. 6:1174-1185.

30. Montarry, J., Corbiere, R., and Andrivon, D. 2007. Is there a trade-off between aggressiveness and overwinter survival in Phytophthora infestans? Funct. Ecol. 21:603-610.

31. Musker, R. 1994. Genetics and population studies of Gaeumannomyces graminis. Ph.D. thesis, University of Birmingham, UK.

32. Nowak, M. A., and Sigmund, K. 2002. Super- and coinfection: The two extremes. Pages 124-137 in: Adaptive Dynamics of Infectious Diseases. In Pursuit of Virulence Management. U. Dieckmann, J. A. J. Metz, M. W. Sabelis, and K. Sigmund, eds. Cambridge University Press, Cambridge.

33. O'Dell, M., Flavell, R. B., and Hollins, T. W. 1992. The classification of isolates of Gaeumannomyces graminis from wheat, rye and oats using restriction fragment length polymorphisms in families of repeated DNA sequences. Plant Pathol. 41:554-562.

34. Olinky, R., Huppert, A., and Stone, L. 2008. Seasonal dynamics and thresholds governing recurrent epidemics. J. Math. Biol. 56:827-839.

35. Rayner, A. D. M., Brasier, C. M., and Moore, D. 1987. Evolutionary biology of the fungi. In: Symp. Br. Mycol. Soc. April 1986. Cambridge University Press, Cambridge.

36. Sarniguet, A., and Lucas, P. 1992. Evaluation of populations of fluorescent pseudomonads related to decline of take-all patch on turfgrass. Plant Soil 145:11-15.

37. van Baalen, M., and Sabelis, M. W. 1995. The dynamics of multiple infection and the evolution of virulence. Am. Nat. 146:881-910.

38. van Boven, M., and Weissing, F. J. 2004. The evolutionary economics of immunity. Am. Nat. 163.

39. van den Bosch, F., Jeger, M. J., and Gilligan, C. A. 2007. Disease control and its selection for damaging plant virus strains in vegetatively propagated staple food crops; a theoretical assessment. Proc. R. Soc. London B 274:11-18.

40. van Molken, T., and Stuefer, J. F. 2008. Virulence in clonal plants: Conflicting selection pressures at work? Evol. Ecol. 22:467-470. 